\title{
High-Angular Splitting Electron Vortex Beams Generated by Topological Defects
}

\author{
Xiaoyan Zhong ${ }^{1}$, ShowShiuan Kao ${ }^{1}$, Jie Lin ${ }^{1}$, Zhenyu Liao ${ }^{1}$, Jing Zhu' ${ }^{1}$, Xiaojing Huang ${ }^{2}$, Rui Zhang ${ }^{3}$, \\ Huolin L. Xin ${ }^{3,4}$ \\ 1. National Center for Electron Microscopy in Beijing, Key Laboratory of Advanced Materials (MOE), \\ State Key Laboratory of New Ceramics and Fine Processing, School of Materials Science and \\ Engineering, Tsinghua University, Beijing, China. \\ ${ }^{2}$. National Synchrotron Light Source II, Brookhaven National Laboratory, Upton, New York, United \\ States. \\ 3. Department of Physics and Astronomy, University of California, Irvine, United States. \\ 4. Center for Functional Nanomaterials, Brookhaven National Laboratory, Upton, New York, United \\ States.
}

Wavefront shaping of transmitted electrons has attracted lots of attentions due to its wide application in materials science and biology. Particularly electron vortex beam carrying an orbital angular momentum can potentially detect magnetic signals at the atomic resolution by theoretical calculations [1, 2]. Up to now there are many approaches to realize the vortex beam with desired topological charges via the predesigned phase plate [3-6]. In 2010, Verbeeck and his colleagues used pitch-fork aperture made in a thinned Pt foil to generate vortex beam [4]. Although the current technology such as focused ion beam and electron beam lithography enables us to fabricate the desired phase plate, e.g. the artificial "Y"-like nanofabricated hologram, for generating electron vortex beams, the spacing in the phase plate can only reach at the nanometer scale due to the limited processing precision. It is challenging to make highangular splitting of electron vortex beams

Fortunately, topological defects in solid-state materials offer a feasible route for the fabrication of phase plates which brings on the wave function of electrons being converted from plane wave to electron vortex by breaking the translational symmetry. We performed a series of experiment on a $\mathrm{NiO}$ sample epitaxial grown on a $\mathrm{SrTiO}_{3}$ substrate to demonstrated how defects bring about the interaction between high-energy electron beam and topological orders [7].

In the case of edge dislocations, an extra plane causes a $2 \pi$ phase-winding topological defect which leads to the same topological pattern as the "Y"-like holograms in a three-beam condition. This equivalency enables us to use it as a "Y"-like hologram. When a plane wave of electron passing through the "Y"-like hologram, it can generate a series of electron vortex beams with increasing order of topological charge. We formed a coherent electron nanobeam in a scanning transmission electron microscope and recorded the far-field transmitted patterns as the beam steps through the edge dislocation core of $\mathrm{NiO}$. Interestingly, the amplitude patterns of the Bragg disks evolve in a similar manner to the evolution of an annular solar eclipse. More importantly, we reconstructed the missing phase information by the ptychographic technique. In other words, we visualized the evolution of phase and amplitude information during the generation of electron vortex beam by topological defects of materials. We demonstrated how atomistic topological defect can convert an electron plane wave into an electron vortex beam, from which the amplitude and phase information can be simultaneously resolved by coherent electron nano-diffraction and electron ptychography. 
Theoretically, the electron vortex beams generated by atomic topological defects provide a three-orderslarge angular separation magnitude and much high collection angle than what traditional nanofabrication technology can offer. The advance will enable the collection of magnetic circular dichroism spectra with high spatial resolution and high efficiency. It might also help us to understand more about the consequence between magnetic interactions and topological defect at the atomic scale [8].

\section{References:}

[1] SM Lloyd et al, Rev. Mod. Phys. 89 (2017), 035004.

[2] DS Negi, JC Idrobo, and J Rusz, Sci. Rep. 8 (2018), 4019.

[3] M Uchida and A Tonomura, Nature 464 (2010), p. 737.

[4] J Verbeeck, H Tian and P Schattschneider, Nature 467 (2010), p. 301.

[5] BJ McMorran et al, Science 331 (2011), p. 192.

[6] V Grillo et al, Nat. Commun. 8 (2017), 15536.

[7] XY Zhong et al, ACS Nano. In press (2019).

[8] This work was financially supported by National key research and development program (2016YFB0700402), National Natural Science Foundation of China (51788104, 11834009 , 51761135131, 51822105, 51671112, 51527803) and National 973 Project of China (2015CB921700, 2015CB654902). This work made use of the resources of the National Center for Electron Microscopy in Beijing. The acquisition of the electron microscopy data was done at and supported by the Center for Functional Nanomaterials, which is a U.S. DOE Office of Science Facility, at Brookhaven National Laboratory under Contract No. DE-SC0012704. R. Z. and H. L. X. are also supported by the set-up funds provided by the University of California, Irvine.

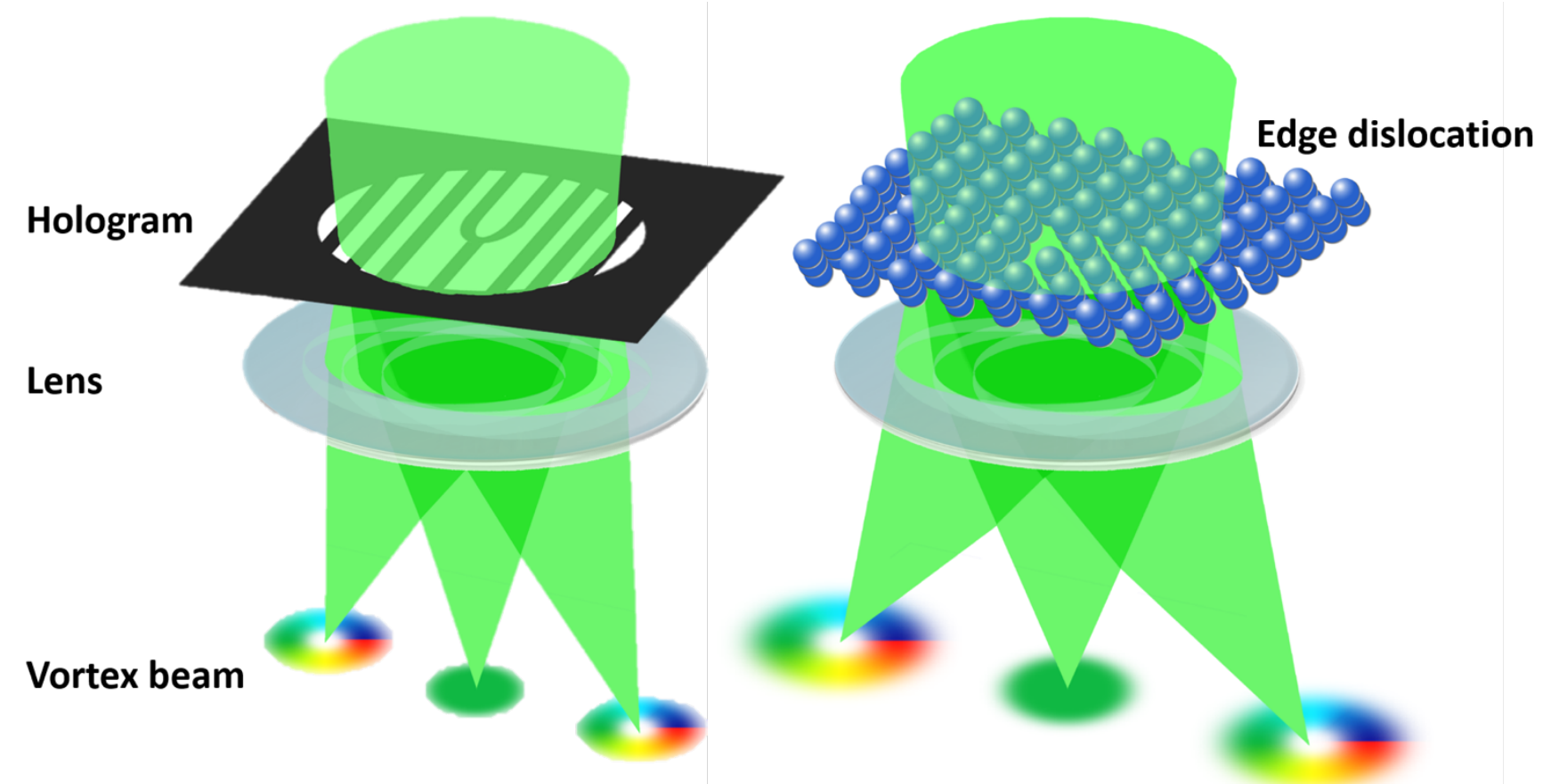

Figure 1. Left. Schematic showing that electrons plane wave being converted to a vortex beam through pitch-fork aperture. Right. Schematic showing that electrons plane wave being converted to a vortex beam by the edge dislocation with a higher angular separation compared to the traditional aperture [7]. 